\title{
The application of color and materials in the theme restaurant design of "desert spring"
}

\author{
Chunbao Zhang \\ Hebei Academy Of Fine Arts, Hebei, 050700 \\ hunter2011@foxmail.com
}

Keywords: Theme restaurant; Color; Material; Design

\begin{abstract}
With the development of science and technology, people's living environment quality requirements gradually increased, people's pursuit of the theme of the restaurant also tend to diversify, and the theme restaurant design color and material use can effectively meet people's subjective needs. When different colors and materials, it will lead to the psychological feelings of empathy.The "desert" theme restaurant is designed to carry out the color and materials for effective collocation and integration, in order to achieve the interaction between the material and color, creating a "desert of spring" atmosphere of the atmosphere, enhance the cultural temperament.
\end{abstract}

\section{Introduction}

Theme restaurant design is based on a certain design method to beautify the restaurant space environment, it can reflect the shape of the restaurant space, the tone of interest and variety of functional requirements [1]. Main restaurant design characteristics are mainly embodied in two aspects of color and decoration materials, only to fully grasp the various colors and various materials properties, the use of the rich expression, mood and spirit, in accordance with the use of environmental conditions and to express spatial atmosphere requires reasonable selection of a desired color and condition, give full play to its strengths, to do only do their best, make the best use of everything, in order to highlight the theme restaurant design features. In this paper, the use of color and materials in the theme restaurant design of "the spring of desert" is discussed in this paper.

Two, the main restaurant overview. Theme restaurant concept. Theme restaurant, is a place of food restaurants through one or more themes for the symbol of the attraction. Theme restaurant in the theme is the central idea of the dining space design, theme culture as the core of the restaurant, combined with the restaurant's furnishings, internal structure, lighting, color and other design [2]. "Hunger breeds discontentment" reflects the food is the most popular gathering place. Theme restaurant after customer observation and imagination, into the expectations of the situation the feeling of be personally on the scene, for example, review a history of years or visit a tribes, or experience a curiosity or stimulation of culture and so on, typical of such restaurants have Wu lin restaurant, the restaurant toilets, old Shanghai restaurant.

Theme restaurant development. In London, in 1971, the first popular theme restaurant, a hard rock restaurant, was founded in London. In 1980, rebuilt in Romania castle in Transylvania Castle Restaurant,on the vampire theme. At home, the theme restaurant in Shanghai opened in the end of the last century, in 2000 in the tropical rain forest restaurant to environmental protection as the theme, in 2001, the opening of the Beijing Zen cool to prison as the theme, such theme restaurants make people find a new way to eat, change the "eat" in people's minds.

Three, the use of color in the theme restaurant design. As the first visual color, the physical stimulation of the human being will have a direct influence on human physiology. The color of people's psychological, causing people's memory and association, so as to arouse people's emotion, is the key to the theme of color. By contrasting colors to achieve the performance and functions of color, some color contrast colorful dazzling, filling gorgeous, and some color contrast is implicit stability, reveals the simple and unadorned. Different colors have different influence on people's emotion and mood [4]. 
(1) Red. Red is the most intense, the most exciting color, the theme of the restaurant in the appropriate use of red can attract people's attention, to mention the color of the entire restaurant. But red is used in restaurant will make the blood increased, the pulse rate, emotional excitement and impulse, so the large red appear at the same time, people are likely to have emotional tension. Therefore, theme restaurants need will be red and dark green, bronze, dark red and other relatively low-key color collocation, so not only can make the red stand out, and will not form contrast over the negative effect. In addition, the red is too strong, so that the overall picture of the restaurant imbalance, so, in the red decoration, should try to use a small area scattered, to create a prominent, the overall harmonious theme restaurant.

(2) White. White represents a lively, pure, romantic, it can not only become the theme of the elegant white color of the main body, and easily and other colors collocation into a rich color of the main body.

White has high brightness, so easily from the background talent showing itself. When you need to use white and other bright colors of collocation, it is appropriate to choose a smaller, more dispersed color type, to reduce the visual impact of white. In addition, the White has a strong reflection of the characteristics, can effectively improve the space, especially the overall environment is in a cold, white is mysterious and elegant. In addition, black and white collocation is pure, but the content is rich.

(3) Yellow. Yellow is a common color, with bright and cheerful personality. Yellow in the restaurant space will cause people's appetite, while feeling warm and rich.

Yellow and orange, red and other warm colors of collocation, is extremely easy to form a harmonious color effect, and with blue, purple and other color collocation, it will form a complementary color contrast. At this point, the need to seriously deal with the depth of color, through appropriate to the middle of some of the color, clever collocation, to form a harmonious theme restaurant screen.

(4) Blue. Blue has a unique quiet temperament, as a piece of blue plants appear in the theme restaurant, people often can be the wave of the sea as a result of the landscape is deeply attracted. Blue used in restaurant space will make people's pulse slow, emotional calm, and feel relaxed, soothing.

Blue overall makes people feel relaxed and quiet, with green or white appears more attractive. Blue and orange or yellow are easy to form a strong color contrast effect, especially in blue and brown orange contrast, the effect is the most obvious.

(5) Purple. Purple has a mysterious, quiet temperament, as a cold color, it is easy to be submerged in a large green or other dark lines.

Purple with similar purple red and blue purple color harmony of form beautiful, luxurious color screen, but if and yellow collocation, often because of the partial characteristics of cold and retired as the background color.

(6) Green. Green is the natural color of plants in nature, and it is also the best medium for the integration of plant color.

In the theme of green restaurant design, you can make full use of the subtle changes in the green tone, create a clear, full of rhythm theme restaurant screen.

The theme restaurant color design focus is the main color of the design, to become the visual center, the main color and background color collocation to coordinate, at the same time, to have a comparison. Theme restaurant color can be divided into a lot of levels, the more levels, the more complex color. Theme restaurant color between different levels of relationship, to respectively, considering the background color and color point, if the background color for the large area of the color, you should use gray tone, and color key for the small area of the color, chroma and lightness to higher than the background color. After the unity of tone, strengthen the intensity of color, that is, through the repetition, rhythm and contrast stressed the theme restaurant need to highlight some of the color effect. Through the color of echo, contact and repeat, can enhance the sense of rhythm and color of color, making the theme restaurant color to achieve unity, so as to form a relatively complete harmony.In the theme restaurant color design, often according to the staff of the 
occupation, age, character or according to the different choice of different colors, to create a different theme restaurant atmosphere, to meet the physiological and psychological needs of customers dining.

Four, the use of materials in the theme restaurant design

\section{Theme restaurant design material characteristics}

Material is the material basis of the theme restaurant design, is the basis for the theme restaurant creativity, which mainly has the following characteristics:

Physical properties of materials". Material itself without any emotion, to make the material of the user of the material cognition emotional resonance, that is when people see a material or touch a material to produce a common psychological feelings. In view of the characteristics of the material, the theme restaurant design, its main task is how clever use of materials to achieve creativity, as well as through the creation of ordinary materials show different emotional atmosphere.

Material performance diversification. Materials not only have the form (original processing), texture (rough and smooth), color (cold and warm, bright and dark) and other physical and chemical properties, and light weight, good strength and anti-corrosion, moisture-proof, fireproof, sound absorption, sound insulation, antistatic, anti aging, and energy-saving, safety, environmental protection, regulation of temperature and humidity etc. performance. The performance of the material is both artistic and scientific and technological. Modern interior decoration materials more and more tend to the green environmental protection, composite materials and finished products and semi-finished products.

The function of the material. Theme restaurant in the use of functional materials, mainly reflected in the use of functional and aesthetic functions. Theme restaurant in the most basic role is to meet the use function, such as dining hall floor and kitchen floor should choose a different floor tiles, kitchen floor tiles choice is to consider the economic benefits, oil, and restaurant lobby is to meet the basic functions should also be combined with restaurant design style, play a certain role in decoration. Landscaping, first of all, the material has a specific decorative features. Under the theme restaurant culture background, the material is used to express the culture or a specific form of carrier. Secondly, the material can create mood and style.

\section{Theme restaurant design of the type of material}

Modern interior decoration materials, not only can improve the indoor environment, so that people enjoy the beauty, but also with the insulation, moisture, fire, sound absorption, sound insulation and other functions, plays a protective structure of the main structure, to extend its service life and meet certain special requirements, is an indispensable material of modern architectural decoration.

There are many different kinds of indoor decoration materials, according to the material classification with plastics, metals, ceramics, glass, wood, inorganic minerals, paints, textiles, stone and other types, classified by function has sound absorption, thermal insulation, waterproof, fireproof, moistureproof and mouldproof, acid and alkali resistant, pollution resistant species. According to the classification of the decorative parts have wall decoration materials, ceiling decoration materials, decoration materials on the ground.

\section{Theme Restaurant Design of the use of materials}

Material is the structure and organization of the material itself, it is the nature of the material. The theme of the restaurant theme design features of a large extent by the constraints of decorative materials, in particular, is subject to the decorative material gloss, texture, pattern, decorative patterns, etc.. A variety of new materials, the main sense of change unpredictably strong to create a space with different psychological feelings.Texture is the material of the material is the visual experience and tactile sensation through the brain to produce a comprehensive treatment of the 
material characteristics of the feeling and impression, which has a material form, color, texture and texture, etc.. The beauty of the material is not only in its pattern, but also in the sense of touch, but in harmony and coordination. The beauty of the material is not in isolation, but in the fusion of other aesthetic elements. In the theme restaurant design interface and the performance of space objects, the right choice and use of materials, so that the material of the material beauty is fully reflected, so as to create a comfortable, harmonious, but also has a unique personality of the theme restaurant space environment [5].

Theme restaurant in the decoration, decoration materials to the very important role, and the beauty of the material texture is very important factors, different materials have different effects, such as the rough stone wall is with a sense of the original power, cement surface is rough sculpture [6]. Often these "expressions" and the theme of the creative link together to express the theme of a certain environment.Although the distribution of decoration is more scattered, a wide variety, but the expression of the theme plays a key role, the style of the decoration often reflect the theme style features. Specific table blanket, calligraphy and painting, sculpture, souvenirs and other, very easy to let diners have intimacy, easier for consumers to have the association, Chujingshengqing. For example, "video" absorb nutrients from the local culture in East Sichuan, extract the main elements from Diaojiao Lou material. Again, surface materials to wood, stone (rag, rubble and stones and pebbles), tiles, paint, through the clever design of the decoration and art to reproduce the beauty of folk customs, simple and natural beauty, arouse memories of the wonderful memories, let a person feel home call home warm.

Five, color and materials in the "desert spring" theme restaurant design application

\section{"spring" theme restaurant lobby space design of the color and the use of materials}

Theme restaurant lobby is an important structure for the guests to leave the first impression, the main function of the performance in guiding and receiving customers, providing leisure or catering services, etc.. The restaurant entrance on to the guests bring distinguished courtesy, its color is an iconic image of the overall style of the restaurant, usually by warm, warm color layout in the first time to bring customers some visual impact. Among them, in the style of contemporary restaurant general through concise small amount of color to highlight the environment in the atmosphere, personality, and the traditional style of the restaurant entrance is more biased to choose warm color gradual change rhythm, to highlight its noble, elegant and unique temperament [7].

"Spring in the desert" theme restaurant choice Brown warm color as the main tone with the white ceiling lamps, coupled with small white energy-saving lighting embellishment, simple and atmospheric, elegant but warm, to customers create a comfortable, pleasant and quiet dining environment, as shown in Figure 1.

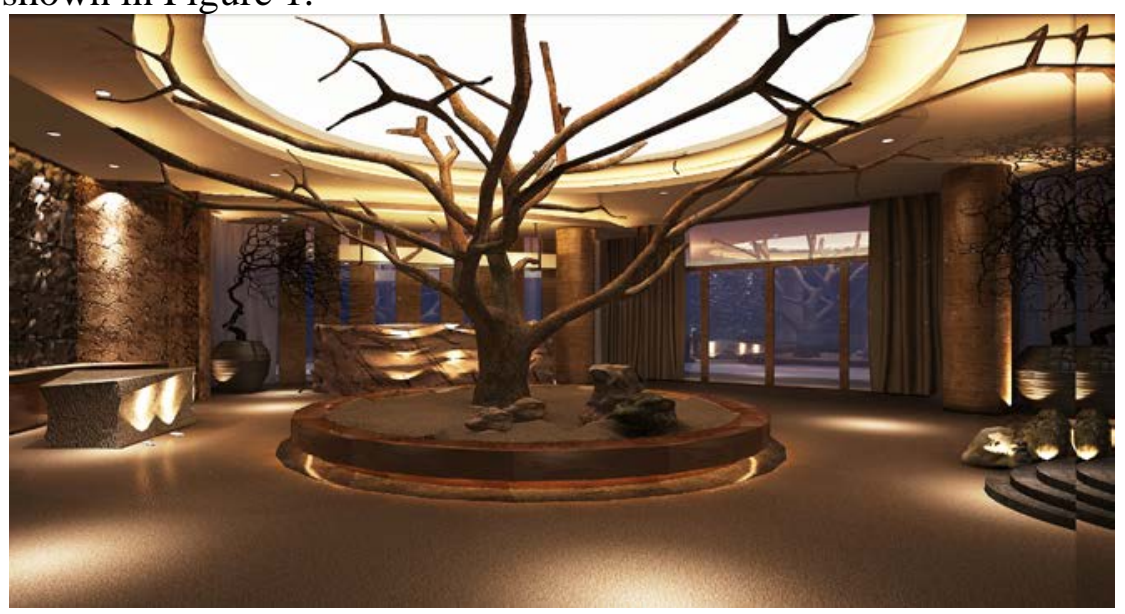

Figure 1 "desert spring" theme restaurant lobby effect

In addition, "spring in the desert" theme restaurant is the central design a tree of a hundred years dead, dead by the simulation of solid wood mosaic,next to deserve to act the role of with rockery, around the rockery stack sand, diners to create a desert edge mood [8]. The white light and the 
wood roof accessories add radiance and beauty to each other reflects the "desert springs," the theme of the restaurant.

\section{"desert spring" theme restaurant design in the dining area of the use of color and materials}

The dining area of the general theme restaurant includes Chinese restaurant and Western restaurant.

The restaurant often to Zhu red, gold, black, yellow and so on noble classical color based, creating a splendid feeling, and restaurant tables and chairs and other furniture to the plain black or delicate brown, furnishings for traditional bronze or porcelain device, the color of the whole environment exudes a strong historical precipitation, when necessary, designers often through use of calligraphy and painting, screen, simple and elegant decoration facilities to ease the heavy gold, red and black color brought visual stimuli in the overall tone to wealth into some implications of scholarly.

And western restaurant color design is more focused on the concise, often used with modern sense of milky white, creamy yellow, light brown color capable, both to increase the guests appetite, and can make whole dining room environment is clean and elegant. In addition, some Western restaurants will use some color gorgeous furniture to render a noble and elegant dining atmosphere, the dining environment is very fit for leisure, communication and other functions need to be.

"The dining area of the desert springs" theme restaurant is divided into the dining area and seat area.

"Spring in the desert" theme restaurant dining area with brown warm colors are solid wood tables and chairs, tables and above to pose a rippling wave decorative ceiling with beads of water dripping like lighting, highlights the theme of "spring in the desert", as shown in Figure 2.

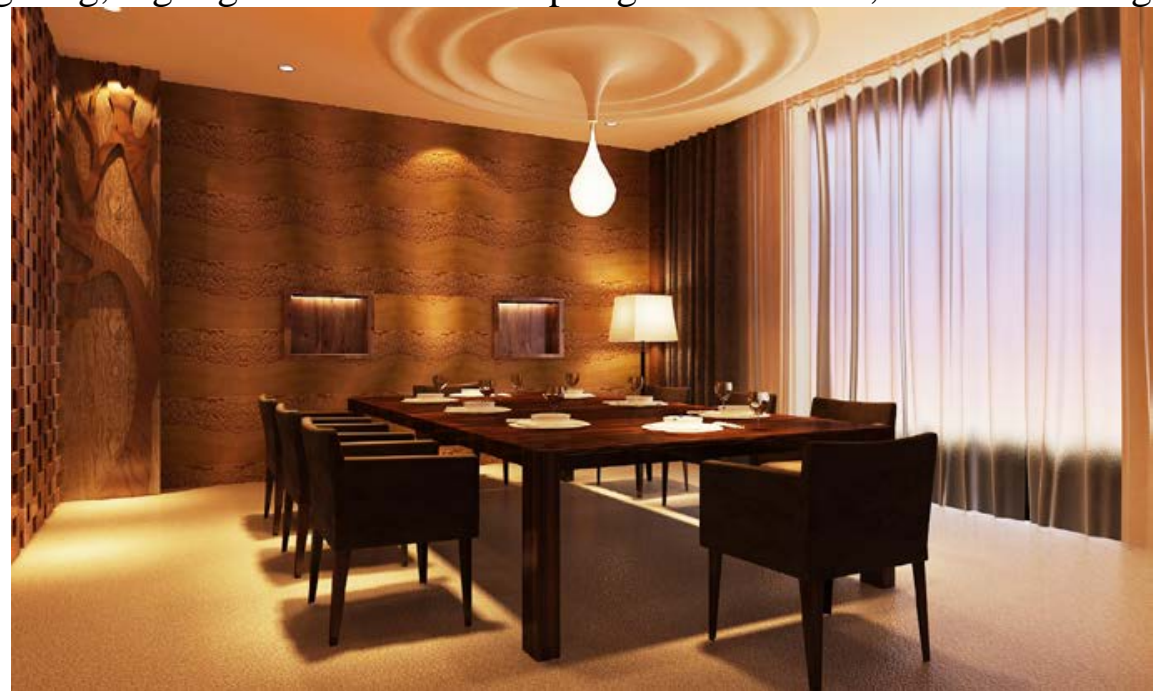

Figure 2 eight. "The spring of the desert", the effect of the dining area on the 2 person dining area

"Spring in the desert" theme restaurant deck areas with brown gray tone of "imitation of the dead Zhuang" tables and chairs, tables and above also to pose a rippling wave decorative ceiling, the same with the beads of water dripping like lighting, and dining area design add radiance and beauty to each other, again highlighting the theme of "spring in the desert", as shown in Figure 3. 


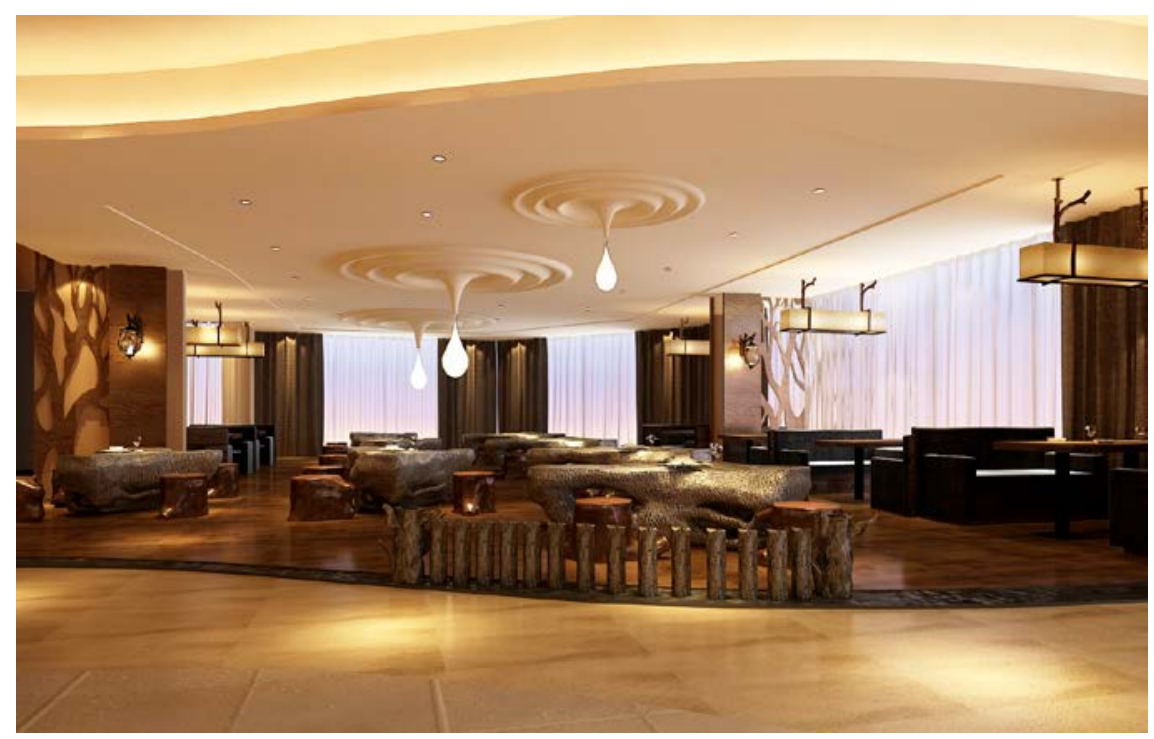

Figure 3 "desert spring" theme restaurant seat area effect

\section{"desert spring" theme restaurant color and material use coordination}

In the theme restaurant in the color of the actual operation is not only according to the color theory is not enough, should be the space in a variety of materials, such as color, furniture, color and lighting color and other factors as a whole to consider, so as to select the theme restaurant and space use features and space overall style coordination of color and material collocation [9].

"Spring in the desert" theme restaurant of colors and materials to coordinate the key lies in design of the channel of the restaurant, this channel will be brownish gray as the main colors, design is in the shape of a smooth line, on both sides of the channel with sporadic lighting, like sand trickling flow Dangqi splash. The channel is above, to streamline the small lights to be modified, and the tone of the channel and the style of unity and coordination, the effect as shown in figure 4.

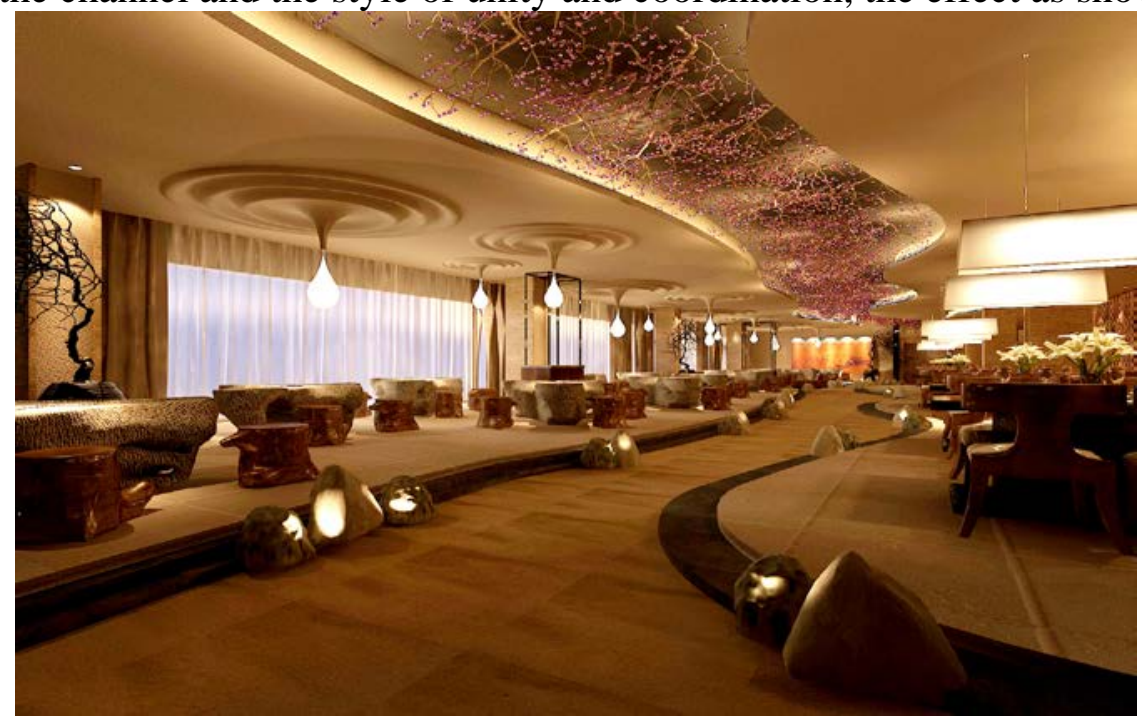

Figure 4 the overall effect of the "desert spring" theme restaurant

\section{Conclusion}

With the continuous improvement of the quality of life, people are increasingly looking forward to a wide variety of theme restaurant dining, color and materials in the theme restaurant design ingenious application, not only to create an elegant and comfortable dining environment, but also to highlight the identity status and cultural quality of dining. To the "desert spring" as the theme of the restaurant design mainly around the use of color and material unity, showing the theme of the restaurant to express the space, the material and color to convey the spirit of the charm. Therefore, 
the use of color and materials to the modern theme restaurant design and the perfect combination of function and beauty is very important [10].

\section{References}

[1] B. Li. The theme restaurant interior design research. Kunming University of Science and Technology, 2013

[2] fortunately, the research on the theme restaurant design method of Soochow University, 2012

[3] Y. Wang. Chongqing theme restaurant development status and Countermeasures of. tourism overview (second half), 2013,02:152-153.

[4] C.J. Liu. Research on the dynamic design of interior color. Chongqing University, 2007

[5] J. Li. The use of modern materials in interior design. China Central Academy of Fine Arts, 2013

[6] Q.L. Wang. Analysis of the use of ground decoration materials in interior design. Guangdong building materials, 2012,11:38-40.

[7] Z.Y. Shen, T.W. Yan. River Garden reception center. interior design and decoration, 2014,12:84-89. 